\title{
Mathematical modelling of TB with the effects of case detection and treatment
}

\author{
S. Athithan • Mini Ghosh
}

Received: 7 May 2013 / Accepted: 18 June 2013 / Published online: 5 July 2013

(C) Springer-Verlag Berlin Heidelberg 2013

\begin{abstract}
In this paper a non-linear mathematical model of tuberculosis with case detection and treatment is proposed and analyzed. The whole population under consideration is divided into four compartments e.g. susceptible, exposed, infected and Recovered to study the transmission dynamics of the tuberculosis. Based on the immunity level, susceptible individuals move to exposed class or directly to infected class once they come into contact with an infective. This has been incorporated through progression rate which could be fast or slow. The equilibria of the model and the basic reproduction number $\mathcal{R}_{0}$ are computed. It is observed that the disease free equilibrium of the model is locally asymptotically stable when $\mathcal{R}_{0}<1$. The model exhibits backward bifurcation under certain restriction on parameters, which gives rise to existence of multiple endemic equilibria for $\mathcal{R}_{0}<1$. This suggests that an accurate estimation of parameters and the level of control measures are required to reduce the infection prevalence of TB in endemic region and just $\mathcal{R}_{0}<1$ is not enough to eliminate the disease from the population. $\mathcal{R}_{0}$ needs to be lowered much below one to confirm the global stability of the disease free equilibrium. Numerical simulation is performed to demonstrate the analytical results. It is found that the increase in the rate of case detection shifts the bacward bifurcation diagram towards right which leads to increase in the threshold value of $\mathcal{R}_{0}$. It is also shown that the treatment is reducing the equilibrium level of infective
\end{abstract}

\section{S. Athithan · M. Ghosh $(\varangle)$}

Department of Mathematics, School of Advanced Sciences,

VIT University Chennai Campus, Vandalur-Kelambakkam Road,

Chennai 600 127, India

e-mail: minighosh@vit.ac.in

S. Athithan

e-mail: athithan1625@gmail.com population. Numerical simulations have been carried out to support the analytic results.

Keywords Tuberculosis - Mathematical model .

Case detection $\cdot$ Stability $\cdot$ Basic reproduction number

\section{Introduction}

Tuberculosis is the second most common cause of death from infectious diseases in developing countries (after those due to HIV/AIDS). Even though this disease is treatable and curable but still it causes nearly one and a half million deaths each year [1]. Even-though total elimination of the disease is not possible due to the difficulty of developing an effective vaccine, the expensive and time-consuming diagnostic process, the necessity of many months of treatment, but we can control the transmission level of the disease. The main task is to provide proper treatment to people infected with TB. And for this first step is case detection. In country like India, lots of TB infected individuals are not aware of their infection and they transmit this disease to others. Sometimes infected individuals give up treatment and go back to work and this also causes new TB infection to people surrounding them. So case detection and proper treatment are very important in controlling the transmission of TB. TB deaths occur mostly in low and middle income countries. Mycobacterium tuberculosis is the bacterial agent which makes the growth of Tuberculosis (TB) in humans. The usual target of this agent is lungs, but can strikes any part of the body such as the kidney, spine, and brain. TB disease can bring to death if not treated properly. If the diagnosis of TB (especially respiratory TB) is postponed or inadequate treatment of cases with latent TB lead to the high infection prevalence of this disease in endemic areas [2]. Mathematical modelling is one of the best tools for the better 
understanding of the transmission dynamics of TB. Many authors have proposed and analyzed simple compartment models for TB. The control and prevention of Tuberculosis using drug chemotherapy in infected individuals had been exhibited in [3]. The efficacy of each drug was explored and observations show that low drug efficacy values result in extension of treatment period. The drug model simulations and analysis show that administration of the recommended first line three-drug regimen normally cures the TB infection. Using the model [3], the authors established that only Isoniazid monotherapy drug treatment, and any combination therapy of two drugs including Isoniazid are potent enough to resolve the TB infection. TB is not only spread by direct contacts with infectives in the population but also indirectly by bacteria which are emitted by infectives in the habitat.

Based on this in [4], authors formulated a model where variable human and bacteria populations are taken into account and it is concluded that due to bacteria in the environment, equilibrium level of the infective population increases. The global properties of a very general tuberculosis model with two differential infectivity is discussed in [5]. The case detection and its effectiveness had been discussed in [6]. Here authors have considered a compartment model, where whole population is divided into subclasses $S, E, I_{1}, I_{2}$ and $R$ where $R$ is the latent class arises due to treatment failure. They considered standard incidence and showed that system exhibit backward bifurcation which makes eradication of TB very tough. In this paper, we formulated similar model as in [6] by considering simple mass action type incidence. But here we divide whole population into only four subclasses, i.e. susceptible, exposed, infected and recovered classes. Our model is much simpler than the model presented in [6] but it exhibits all the complexities discussed in the paper [6]. This paper is organized as follows: Sect. 2 describes the basic model, Sect. 3 describes the existence of equilibria and stability analysis. In Sect. 4, we conduct some numerical computations to support our analytical findings and finally we summarize our results in Sect. 5 .

\section{The model}

Here an SEIR model is presented to study the transmission dynamics of TB by dividing the whole population into four different classes e.g. susceptible class, exposed class, TB infected class and the class of recovered individuals (Susceptible-Exposed-Infected-Recovered). Here we discuss both treatment and case detection. Let $S(t), E(t), I(t)$ and $R(t)$ be the fractions of the susceptible individuals, the exposed (latent) individuals, the TB infectious individuals and the treated/recovered individuals in the population, respectively, at time t. Hence, $N=S+E+I+R$ is the total population. It is assumed that the total population is varying

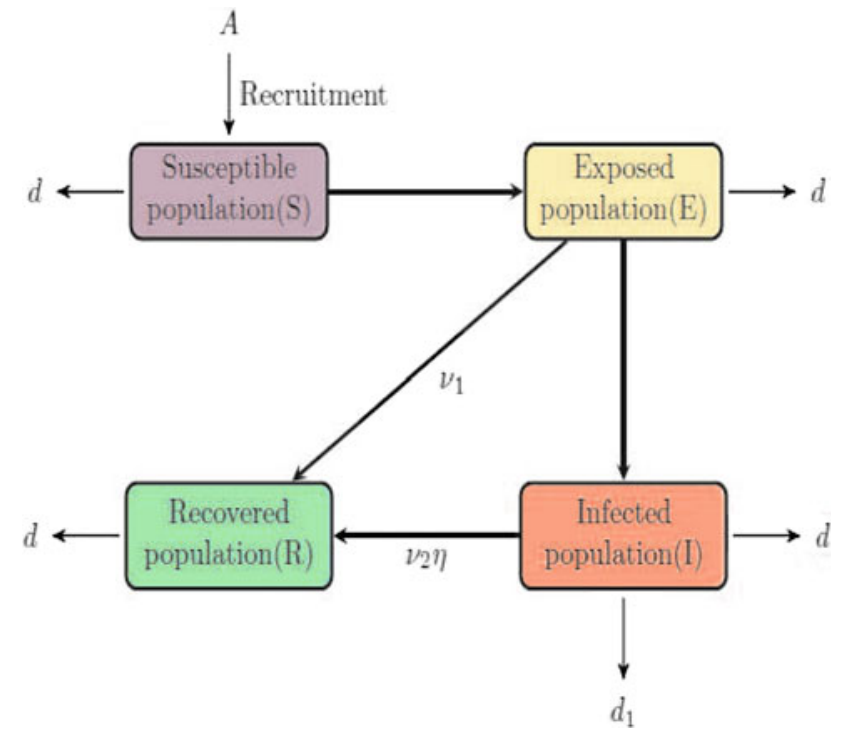

Fig. 1 Transfer diagram of the model (1)

and homogeneously mixed i.e., all people are equally likely to be infected by the infectious individuals in a case of contact. Also it is assumed that a fraction $\eta$ of infected individuals is identified with TB so they are under treatment. So transmission of TB due to these detected TB patients will be less compared to those infected individuals who are not identified with TB. Also immunity level of individuals vary, so it is assumed that a fraction $0<p<1$ of the individuals with new infection develops TB fast and directly joins infected class where as $(1-p)$ fraction of individuals with new infection moves to exposed class first and then gradually moves to infected class. Again if exposed individuals come into contact with infected individuals, they become infectious. Also there is disease progression and exposed individuals become infected after some time if not treated. The transfer diagram of the model is described in Fig. 1. Keeping the above in mind and by considering simple mass action interaction, a mathematical model is proposed as follows:

$$
\begin{aligned}
S^{\prime}= & A-d S-\left[\alpha_{1} \eta+\alpha_{2}(1-\eta)\right] I S, \\
E^{\prime}= & (1-p)\left[\alpha_{1} \eta+\alpha_{2}(1-\eta)\right] I S \\
& -\beta E I-\left(d+v_{1}+\theta\right) E, \\
I^{\prime}= & p\left[\alpha_{1} \eta+\alpha_{2}(1-\eta)\right] I S+\beta E I \\
& -\left(d+d_{1}+v_{2} \eta\right) I+\theta E, \\
R^{\prime}= & v_{1} E+v_{2} \eta I-d R .
\end{aligned}
$$

The parameters used in the model (1) are described in Table 1.

The model (1) can be further simplified as follows:

$$
\begin{aligned}
S^{\prime} & =A-d S-k_{1} I S \\
E^{\prime} & =(1-p) k_{1} I S-\beta E I-k_{2} E \\
I^{\prime} & =p k_{1} I S+\beta E I-k_{3} I+\theta E \\
R^{\prime} & =v_{1} E+v_{2} \eta I-d R
\end{aligned}
$$


Table 1 Description of parameters

\begin{tabular}{lll}
\hline Parameter & Description & Values \\
\hline$A$ & Recruitment rate \\
$d$ & Natural death rate \\
$\eta$ & Case detection rate \\
$\alpha_{1}$ & Rate of transmission(det.) \\
$\alpha_{2}$ & Rate of transmission(undet.) \\
$p$ & Rate of fast progression to infection \\
$\beta$ & Contact rate b/w E and I \\
$\theta$ & Rate of progression to I from E \\
$v_{1}, v_{2}$ & Recovery rates due to treatment \\
$d_{1}$ & Death rate due to infection \\
\hline
\end{tabular}

where $k_{1}=\left[\alpha_{1} \eta+\alpha_{2}(1-\eta)\right], k_{2}=\left(d+v_{1}+\theta\right), k_{3}=$ $\left(d+d_{1}+v_{2} \eta\right)$.

The suitable domain for the model is given by

$\mathcal{D}=\left\{(S, E, I, R) \in R_{\geq 0}^{4} ; \quad S+E+I+R<A / d\right\}$

and it can be shown that the set $\mathcal{D}$ is a positively invariant set and a global attractor of this system. That is, any phase trajectory initiated anywhere in the non-negative region $R_{\geq 0}^{4}$ of the phase space eventually enters the region $\mathcal{D}$ and remains in $\mathcal{D}$ thereafter.

Here

$N^{\prime}=A-d N-d(E+I+R)-d E-\left(d+d_{1}\right) I-d R$

is the rate of change of the total population $N$. It is observed that the right hand side of the above equality is bounded by $\frac{A}{d}$, a standard comparison theorem can be used to show that

$N(t) \leq N(0) e^{-d t}+\frac{A}{d}\left(1-e^{-d t}\right)$.

If $N(0) \leq \frac{A}{d}$, then $N(t) \leq \frac{A}{d}$. Thus, $\mathcal{D}$ is a positively invariant set under the system described in (2). Hence no solution path leave through any boundary of $\mathcal{D}$. The right hand side of (2) is smooth, hence the initial value problem has a unique solution that exists on maximal intervals [7]. Since paths cannot leave $\mathcal{D}$, solutions remain non-negative for non-negative initial conditions; solutions exist for all positive time. Thus, the model (1) is mathematically and epidemiologically well posed [7].

\section{Equilibria and stability analysis}

3.1 Basic reproduction number and local stability of disease-free equilibrium(DFE)

The model (1) has a DFE given by
$E_{0}=\left(S^{0}, E^{0}, I^{0}, R^{0}\right)=\left(\frac{A}{d}, 0,0,0\right)$.

The stability of $E_{0}$ can be established using the next generation operator method on the system (1). Using the notations in [8], the matrices $F$ and $V$, for the new infection terms and the remaining transfer terms respectively, are given by

$$
\begin{aligned}
& F=\left(\begin{array}{cc}
0 & (1-p) k_{1} S^{0} \\
0 & p k_{1} S^{0}
\end{array}\right) \text { and } V=\left(\begin{array}{cc}
k_{2} & 0 \\
-\theta & k_{3}
\end{array}\right) \\
& F V^{-1}=\left(\begin{array}{cc}
\frac{\theta(1-p) k_{1} S^{0}}{k_{2} k_{3}} & \frac{(1-p) k_{1} S^{0}}{k_{3}} \\
\frac{\theta p k_{1} S^{0}}{k_{2} k_{3}} & \frac{p k_{1} S^{0}}{k_{3}}
\end{array}\right) \\
&=\left(\begin{array}{cc}
\frac{\theta(1-p) k_{1} A}{d k_{2} k_{3}} & \frac{(1-p) k_{1} A}{d k_{3}} \\
\frac{\theta p k_{1} A}{d k_{2} k_{3}} & \frac{p k_{1} A}{d k_{3}}
\end{array}\right)
\end{aligned}
$$

Thus the basic reproduction number $\mathcal{R}_{0}$ which is the spectral radius of the matrix $F V^{-1}$ is given by

$\mathcal{R}_{0}=\frac{\left[(1-p) \theta+p k_{2}\right] k_{1} A}{d k_{2} k_{3}}$

The following result follows from Theorem 2 of Ref. [8].

Theorem 1 The DFE, $E_{0}$ of the model (1) is locally asymptotically stable if $\mathcal{R}_{0}<1$ and unstable if $\mathcal{R}_{0}>1$.

The threshold quantity $\mathcal{R}_{0}$ is the basic reproduction number for the TB model. Biologically speaking, Theorem 1 implies that TB can be eliminated from the community (when $\left.\mathcal{R}_{0}<1\right)$ if the initial sizes of the sub-populations of the model are in the basin of attraction of $E_{0}$.

From Theorem 1, it was established that if the disease free equilibrium exists, it is locally asymptotically stable if and only if $\mathcal{R}_{0}<1$. However, the disease free equilibrium may not be globally asymptotically stable even if $\mathcal{R}_{0}<1$. It is possible for $\mathcal{R}_{0}<1$ and there could be the possibility of backward bifurcation (bi-stability) when a stable endemic equilibrium may co-exist with the DFE. To find the conditions for the existence of the endemic equilibrium for the model (1), denoted by $E_{1}=\left(S^{*}, E^{*}, I^{*}, R^{*}\right)$, the equations in (2) are solved in terms of the infection at steady state $\left(I^{*}\right)$, which must satisfy the following polynomial,

$f\left(I^{*}\right)=A_{1}\left(I^{*}\right)^{2}+B_{1} I^{*}+C_{1}=0$

where $A_{1}=\beta k_{1} k_{3}$,

$B_{1}=\beta d k_{3}+k_{1} k_{2} k_{3}-\beta k_{1} A$ and

$C_{1}=d k_{2} k_{3}-\theta(1-p) k_{1} A-p k_{1} k_{2} A=d k_{2} k_{3}\left(1-\mathcal{R}_{0}\right)$.

The endemic equilibrium in terms of $I^{*}$ is given by

$S^{*}=\frac{A}{d+k_{1} I^{*}}, \quad E^{*}=\frac{(1-p) k_{1} I^{*} S^{*}}{\beta I^{*}+k_{2}}, \quad R^{*}=\frac{\nu_{1} E^{*}+\nu_{2} \eta I^{*}}{d}$

The following cases to be considered (depending on the signs of $B_{1}$ and $C_{1}$, since $A_{1}$ is positive) to study the number of positive roots of $f\left(I^{*}\right)=0$. 
Case 1 Suppose that $\beta=0$ so that we do not have exogenous re-infection, then $A_{1}=0, B_{1}>0$ and the quadratic equation in (3.2) becomes linear in $I^{*}=\frac{C_{1}}{B_{1}}$. Hence, there will be a unique positive root if $C_{1}<0$. In this case, the system has a unique (stable) endemic equilibrium if and only if $C_{1}<0$ which occurs only when $\mathcal{R}_{0}>1$; hence backward bifurcation is not possible in this case.

Case 2 If $B_{1}<0$ and $C_{1}=0$ or $B_{1}^{2}-4 A_{1} C_{1}=0$, then $f\left(I^{*}\right)=0$ has one positive root which means that the system has a unique endemic equilibrium.

Case 3 If $C_{1}>0, B_{1}<0$ and $B_{1}^{2}-4 A_{1} C_{1}>0$, then $f\left(I^{*}\right)=0$ has two positive roots which implies that the system has two endemic equilibria.

The results are summarized in the following lemma.

Lemma 1 The number of positive endemic equilibria of model 2.1 is summarized as follows:

1. If $C_{1}<0$ which occurs only when $\mathcal{R}_{0}>1$, the system has a unique endemic equilibrium.

2. If $B_{1}<0$ and $C_{1}=0$ or $B_{1}^{2}-4 A_{1} C_{1}=0$, then $f\left(I^{*}\right)=$ 0 , the system has exactly one endemic equilibrium.

3. If $C_{1}>0, B_{1}<0$ and $B_{1}^{2}-4 A_{1} C_{1}>0, \mathcal{R}_{0}<1$, the system has exactly two endemic equilibria (say $E_{1}$ and $E_{2}$ ).

4. Otherwise there is no endemic equilibria, i.e. when $A_{1} C_{1}>0, B_{1}>0$.

If $\mathcal{R}_{0}<1$, we can derive

$$
\mathcal{R}_{0}^{c}=1-\frac{B_{1}^{2}}{4 A_{1} d k_{2} k_{3}}
$$

such that $B_{1}^{2}-4 A_{1} C_{1}>(=$ or $<) 0$ if $\mathcal{R}_{0}>(=$ or $<) R_{0}^{c}$, where $\mathcal{R}_{0}^{c}$ is the backward bifurcation point and it can be shown that backward bifurcation occurs for values of $\mathcal{R}_{0}$ in the range $\mathcal{R}_{0}^{c}<\mathcal{R}_{0}<1$ ( see point 3 in Lemma.1).

\subsection{Bifurcation analysis}

Let $S=x_{1}, E=x_{2}, I=x_{3}, R=x_{4}$, so that $N=x_{1}+x_{2}+$ $x_{3}+x_{4}$; hence the model (2) is re-written in the form

$$
\begin{aligned}
& x_{1}^{\prime}=f_{1}=A-d x_{1}-k_{1} x_{3} x_{1} \\
& x_{2}^{\prime}=f_{2}=(1-p) k_{1} x_{3} x_{1}-\beta x_{2} x_{3}-k_{2} x_{2} \\
& x_{3}^{\prime}=f_{3}=p k_{1} x_{3} x_{1}+\beta x_{2} x_{3}-k_{3} x_{3}+\theta x_{2} \\
& x_{4}^{\prime}=f_{4}=v_{1} x_{2}+v_{2} \eta x_{3}-d x_{4}
\end{aligned}
$$

The Jacobian of the system (6) at DFE is given by

$$
J\left(E_{0}\right)=\left(\begin{array}{cccc}
-d & 0 & \frac{-k_{1} A}{d} & 0 \\
0 & -k_{2} & \frac{(1-p) k_{1} A}{d} & 0 \\
0 & \theta & \frac{p k_{1} A}{d}-k_{3} & 0 \\
0 & v_{1} & v_{2} \eta & -d
\end{array}\right)
$$

Consider the case when $\mathcal{R}_{0}=1$. Suppose that $p=p *$ is chosen as a bifurcation parameter. Solving for $p$ from $\mathcal{R}_{0}=1$ gives

$p=p *=\frac{\theta A k_{1}+d k_{2} k_{3}}{A k_{1}\left(k_{2}-\theta\right)}$.

Using the following theorem from Refs. [8,9] and followed in [6] reproduced below for convenience, one will be able to determine whether or not the system (6) exhibits backward bifurcation at $\mathcal{R}_{0}=1$.

Theorem 2 Consider the following general system of ordinary differential equations with a parameter $\phi$

$\frac{d x}{d t}=f(x, \phi)$,

$f: \mathbb{R}^{n} \times \mathbb{R} \rightarrow \mathbb{R}$

and

$f \in \mathbb{C}^{2}\left(\mathbb{R}^{n} \times \mathbb{R}\right)$,

where 0 is an equilibrium point of the system (i.e. $f(0, \phi) \equiv$ 0 for all $\phi$ ) and

1. $A=D_{x} f(0,0)=\left(\frac{\partial f_{i}}{\partial x_{j}}(0,0)\right)$ is the linearization matrix of the system around the equilibrium 0 with $\phi$ evaluated at 0 ;

2. Zero is a simple eigenvalue of $A$ and other eigenvalues of $A$ have negative real parts;

3. Matrix A has a right eigenvector $w$ and a left eigenvector $v$ corresponding to the zero eigenvalue.

Let $f_{k}$ be the $k^{\text {th }}$ component of $f$ and

$a=\sum_{k, i, j=1}^{n} v_{k} w_{i} w_{j} \frac{\partial^{2} f_{k}}{\partial x_{i} \partial x_{j}}(0,0)$,

$b=\sum_{k, i=1}^{n} v_{k} w_{i} \frac{\partial^{2} f_{k}}{\partial x_{i} \partial \phi}(0,0)$,

then the local dynamics of the system around the equilibrium point 0 is totally determined by the signs of $a$ and $b$. Particularly, if $a>0$ and $b>0$, then a backward bifurcation occurs at $\phi=0$.

\subsubsection{Eigenvalues of $J_{p *}$}

For the case $\mathcal{R}_{0}=1$, it can be shown that the Jacobian of system (6) at $p=p *$, denoted by $J_{p *}$ has a right eigenvector given by $w=\left[w_{1}, w_{2}, w_{3}, w_{4}\right]^{T}$, where 
$w_{1}=\frac{-k_{1} A}{d^{2}} w_{3}$,

$w_{2}=\frac{(1-p *) k_{1} A}{d k_{2}} w_{3}<0$,

$w_{3}=w_{3}<0$,

$w_{4}=\frac{v_{1} w_{2}+v_{2} \eta w_{3}}{d}<0$

Further, $J_{p *}$ has a left eigenvector given by $v=\left[v_{1}, v_{2}\right.$, $\left.v_{3}, v_{4}\right]$, where

$v_{1}=0$,

$v_{2}=\frac{\theta v_{3}+v_{1} v_{4}}{k_{2}}=\frac{\theta v_{3}}{k_{2}}>0$,

$v_{3}=v_{3}>0$,

$v_{4}=0$.

\section{Computations of $a$ and $b$ :}

For the system (6), the associated non-zero partial derivatives are given by

$\frac{\partial^{2} f_{2}}{\partial x_{1} \partial x_{3}}=\left(1-p^{*}\right) k_{1}$,

$\frac{\partial^{2} f_{2}}{\partial x_{2} \partial x_{3}}=-\beta$

$\frac{\partial^{2} f_{3}}{\partial x_{1} \partial x_{3}}=p^{*} k_{1}$

$\frac{\partial^{2} f_{3}}{\partial x_{2} \partial x_{3}}=\beta$

It follows from the above expressions that

$$
\begin{aligned}
a= & v_{2} \sum_{i, j=1}^{4} w_{i} w_{j} \frac{\partial^{2} f_{2}}{\partial x_{i} \partial x_{j}}+v_{3} \sum_{i, j=1}^{4} w_{i} w_{j} \frac{\partial^{2} f_{3}}{\partial x_{i} \partial x_{j}} \\
= & 2 v_{2} w_{3}\left\{w_{1}\left[\left(1-p^{*}\right) k_{1}\right]-w_{2} \beta\right\} \\
& +2 v_{3} w_{3}\left\{w_{1} p k_{1}+w_{2} \beta\right\}>0
\end{aligned}
$$

if $\beta>\frac{w_{1} k_{1}\left[\left(1-p^{*}\right) v_{2}+p^{*} v_{3}\right]}{w_{2}\left(v_{3}-v_{2}\right)}$ with $w_{2}\left(v_{3}-v_{2}\right)>0$.

For the sign of $b$, it is shown that the associated non-zero partial derivatives are

$$
\begin{aligned}
& \frac{\partial^{2} f_{2}}{\partial x_{3} \partial p^{*}}=\frac{-k_{1} A}{d}, \\
& \frac{\partial^{2} f_{3}}{\partial x_{3} \partial p^{*}}=\frac{k_{1} A}{d},
\end{aligned}
$$

so that,

$$
\begin{aligned}
b & =v_{2} \sum_{i=1}^{4} w_{i} \frac{\partial^{2} f_{2}}{\partial x_{i} \partial p *}+v_{3} \sum_{i=1}^{4} w_{i} \frac{\partial^{2} f_{3}}{\partial x_{i} \partial p^{*}} \\
& =v_{2}\left[w_{3}\left(\frac{-k_{1} A}{d}\right)\right]+v_{3}\left[w_{3}\left(\frac{k_{1} A}{d}\right)\right] \\
& =w_{3}\left(\frac{k_{1} A}{d}\right)\left[v_{3}-v_{2}\right]
\end{aligned}
$$

From these we get $b>0$ only if $v_{2}>v_{3}$. Thus we established the following result.

Theorem 3 If

$\beta>\frac{w_{1} k_{1}\left[\left(1-p^{*}\right) v_{2}+p^{*} v_{3}\right]}{w_{2}\left(v_{3}-v_{2}\right)}=\beta_{0}$

with $w_{2}\left(v_{3}-v_{2}\right)>0$ and $v_{3}>v_{2}$, then the model (6) undergoes a backward bifurcation which occurs at $\mathcal{R}_{0}=1$.

\subsection{Global stability of DFE}

It is important to see that from the inequality (7), the model cannot undergo backward bifurcation if $\beta=0$. More precisely, this study reveals that exogenous reinfection is essential for the occurrence of backward bifurcation, as seen from [10]. Thus backward bifurcation will occur if $\mathcal{R}_{0}^{c}<\mathcal{R}_{0}<1$ and $\beta>\beta_{0}$ (as shown in Sec. 3.1 and as stated in Theorem 3).

Now we can prove the global stability of the DFE.

Theorem 4 The disease-free equilibrium $E_{0}$ of model (2) without exogenous reinfection (i.e. when $\beta=0$ ) is globally asymptotically stable if $\mathcal{R}_{0}<1$.

Proof We can prove the global stability of the DFE using the comparison theorem(see Ref. [11], p. 31). Re-writing the equations for the infected compartments in (2), we have

$$
\left(\begin{array}{c}
E^{\prime} \\
I^{\prime}
\end{array}\right)=(F-V)\left(\begin{array}{c}
E \\
I
\end{array}\right)-\left(\begin{array}{c}
(1-p) k_{1} I\left[S^{0}-S\right] \\
p k_{1} I\left[S^{0}-S\right]
\end{array}\right)
$$

where $F$ and $V$ are as defined in Sec. 3.1. Since $S \leq S_{0}=\frac{A}{d}$ for all $t>0$, it follows that

$$
\left(\begin{array}{c}
E^{\prime} \\
I^{\prime}
\end{array}\right) \leq(F-V)\left(\begin{array}{c}
E \\
I
\end{array}\right)
$$

Since all the eigenvalues of the matrix $F-V$ have negative real parts (this comes from the local stability results in Lemma 1 in [8]), then system (2) is stable whenever $\mathcal{R}_{0}<1$. So, $(E, I) \rightarrow(0,0)$ as $t \rightarrow \infty$. By the comparison theorem, it follows that $(E, I) \rightarrow(0,0)$ and $S \rightarrow \frac{A}{d} t \rightarrow \infty$. Then $(S, E, I, R) \rightarrow E_{0}$ as $t \rightarrow \infty$. So, $E_{0}$ is globally asymptotically stable for $\mathcal{R}_{0}<1$ when $\beta=0$.

\subsection{Local stability of endemic equilibrium (EE)}

Theorem 5 The endemic equilibrium of model (2) is locally asymptotically stable when $a_{2} a_{1}-a_{0}>0$, where $a_{2}, a_{1}$ and $a_{0}$ are given in the proof of the theorem.

Proof The variational matrix, $M^{*}$ corresponding to the endemic equilibrium point is given by

$$
M^{*}=\left(\begin{array}{ccc}
n_{11} & 0 & n_{13} \\
n_{21} & n_{22} & n_{23} \\
n_{31} & n_{32} & n_{33}
\end{array}\right)
$$


where, $n_{11}=-\left(d+k_{1} I^{*}\right), n_{13}=-k_{1} S^{*}$,

$n_{21}=(1-p) k_{1} I^{*}, n_{22}=-\left(\beta I^{*}+k_{2}\right), n_{23}=(1-$ p) $k_{1} S^{*}-\beta E^{*}$,

$n_{31}=p k_{1} I^{*}, n_{32}=\beta I^{*}+\theta, n_{33}=p k_{1} S^{*}+\beta E^{*}-k_{3}$,

We will get three eigen values from the variational matrix, which are the solutions of the polynomial $\lambda^{3}+a_{2} \lambda^{2}+a_{1} \lambda+$ $a_{0}=0$,

where,

$$
\begin{aligned}
a_{2}= & -\left(n_{11}+n_{22}+n_{33}\right), \\
a_{1}= & {\left[\left(n_{22} n_{33}-n_{23} n_{32}\right)+\left(n_{11} n_{33}-n_{31} n_{13}\right)\right.} \\
& \left.+\left(n_{11} n_{22}-0\right)\right], \\
a_{0}= & -\left[n_{11}\left(n_{22} n_{33}-n_{23} n_{32}\right)-0\right. \\
& \left.+n_{13}\left(n_{21} n_{32}-n_{31} n_{22}\right)\right] .
\end{aligned}
$$

By using Routh-Hurwitz criteria, the EE will be locally asymptotically stable if the following conditions are satisfied.

$a_{2}>0$ and $\left|\begin{array}{cc}a_{2} & a_{0} \\ 1 & a_{1}\end{array}\right|>0$.

Here obviously $a_{2}>0$, so EE is locally asymptotically stable if other inequality is satisfied.

\section{Numerical simulation}

The system (2) is simulated for various set of parameters. First we consider following set of parameters for which $R_{0}$ is greater than $R_{0}^{c}$ and less than 1 .

$A=18, \quad d=0.075, \quad \alpha_{1}=0.001, \quad \alpha_{2}=0.003$,

$p=0.235, \quad \eta=0.57, \quad \beta=0.005$,

$\nu_{1}=0.08, \quad v_{2}=0.08, \quad d_{1}=0.032, \quad \theta=0.0001$.

For this set of parameter we get two endemic equilibria

$E_{1}(188.94783,30.46408,10.89483,39.11907)$

and

$$
E_{2}(127.266,30.502,35.718,54.253)
$$

and the disease free equilibrium point $E_{0}(240,0,0,0)$. This fact is demonstrated by S-I phase plane in Fig. 2, where $E_{0}$ and $E_{2}$ are locally asymptotically stable and the equilibrium point $E_{1}$ is unstable. It is more clear from the bifurcation diagrams (see Fig. 3). Figure 3 is obtained by considering the fast progression rate of disease $p$ as the bifurcation parameter. The horizontal axis is labelled with the appropriate value of the reproduction number $\mathcal{R}_{0}$ corresponding to this bifurcation parameter $p$. It is observed that when the reproduction number $\mathcal{R}_{0}$ is between 0 to $\mathcal{R}_{0}^{c}$, the infection free equilibrium alone is stable, for $\mathcal{R}_{0}^{c}<\mathcal{R}_{0}<1$ we have

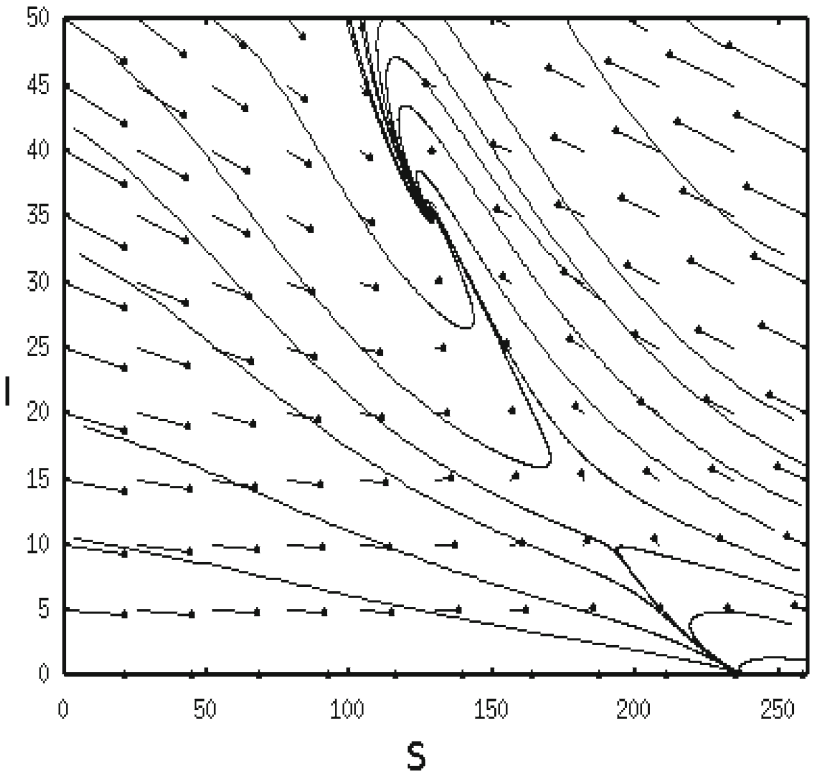

Fig. 2 S-I phase plane showing the bistability

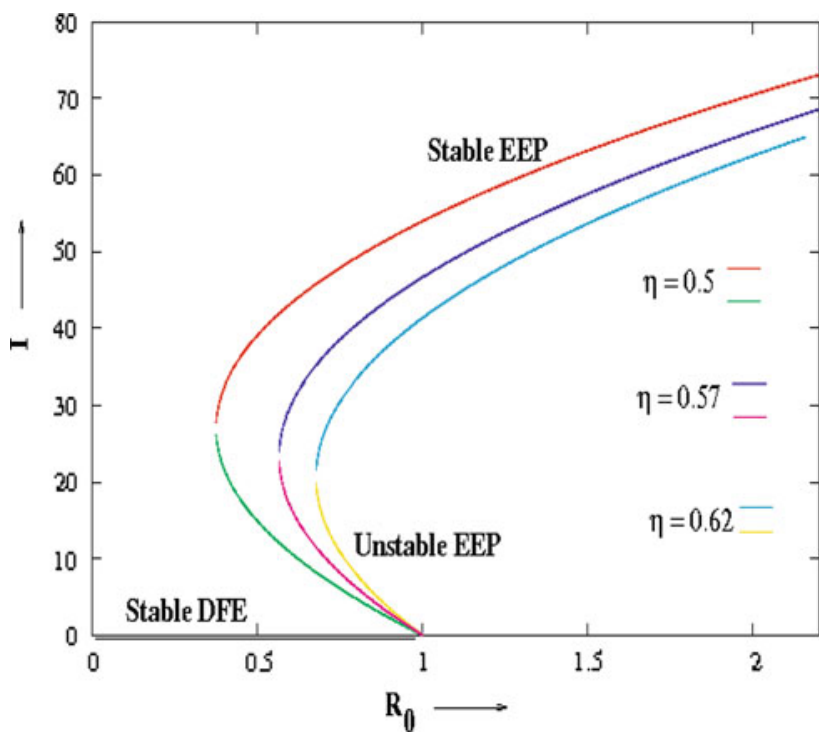

Fig. 3 Backward bifurcation plot of I

bi-stability where either the infection free equilibrium is stable (or) the endemic equilibrium is stable. To see the effect of case detection rate $\eta$ bifurcation diagram is drawn for different values of $\eta$. Figure 3 shows that the case detection plays a vital role in the transmission dynamics of TB. It can be seen that with the increase in the case detection rate the bifurcation diagram shifts towards right implying the increase in

$R_{0}^{c}$ value, which means that if case detection rate is large enough then we will not get backward bifurcation. And in this situation $R_{0}<1$ will be sufficient to eliminate the disease from the population. And for this case, increasing the probability of detection reduces the reproduction number, and 
hence the number of new infections; the critical detection level corresponds to $\mathcal{R}_{0}=1$. Figure 4 , is showing the variation of , $E, I, R$ with time for a different set of parameters for which $\mathcal{R}_{0}=2.63$. Figure 5 is demonstrating the stability of disease free equilibrium point $E_{0}$ for the following set of parameters:

$A=18, \quad d=0.075, \quad \alpha_{1}=0.001, \quad \alpha_{2}=0.003$,

$p=0.4, \quad \eta=0.8, \quad \beta=0.005$,

$v_{1}=0.08, \quad v_{2}=0.08, \quad d_{1}=0.032, \quad \theta=0.0001$.

For this set of parameters $\mathcal{R}_{0}=0.786$ and disease free equilibrium is stable. Here we do not have backward bifur-

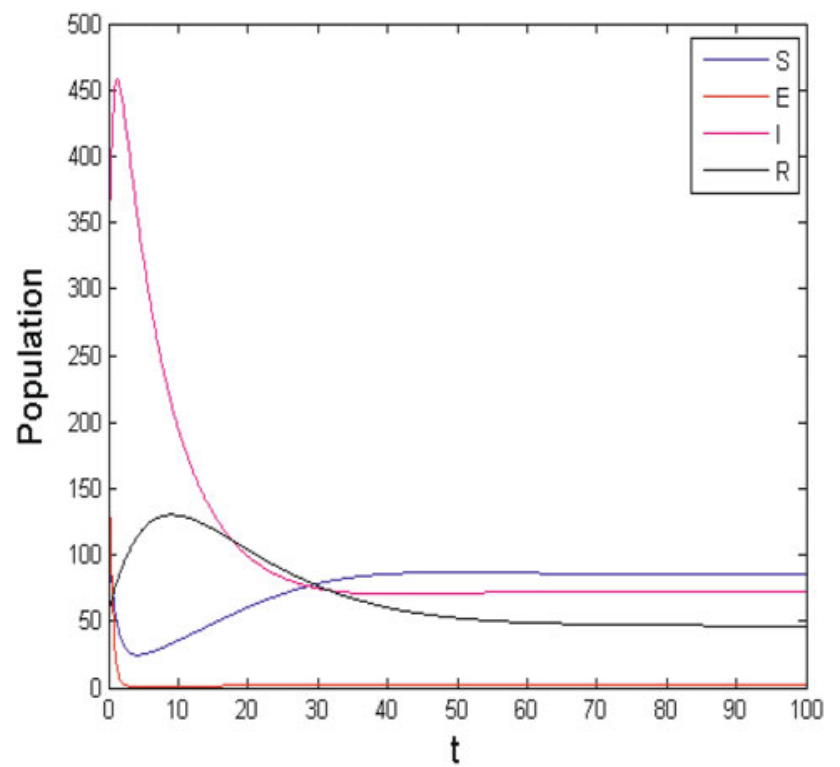

Fig. 4 Variation of $S, E, I, R$ with time for $\mathcal{R}_{0}>1$

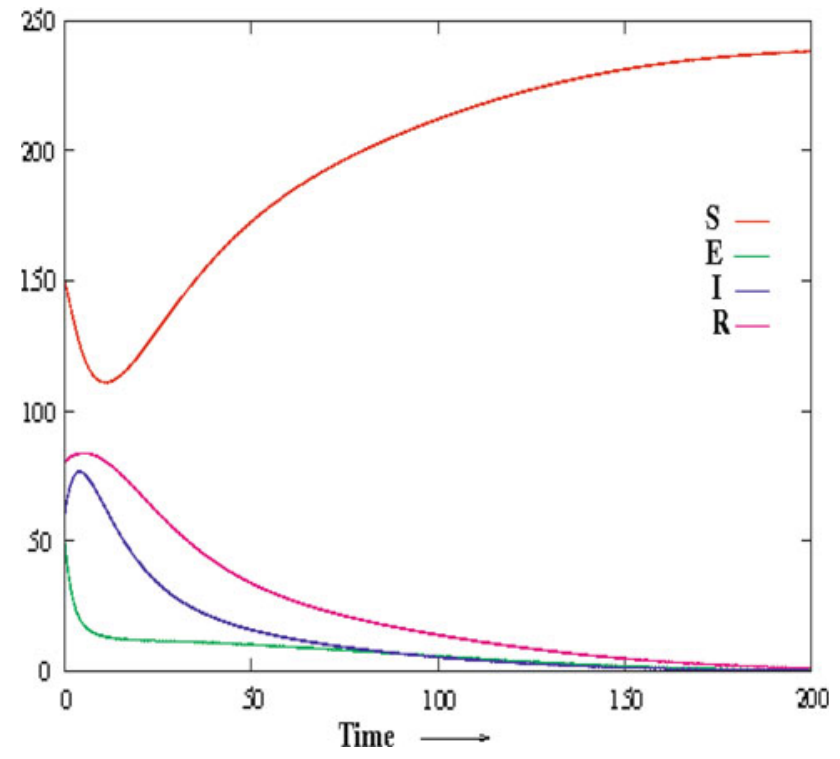

Fig. 5 Variation of $S, E, I, R$ with time for $\mathcal{R}_{0}<1$

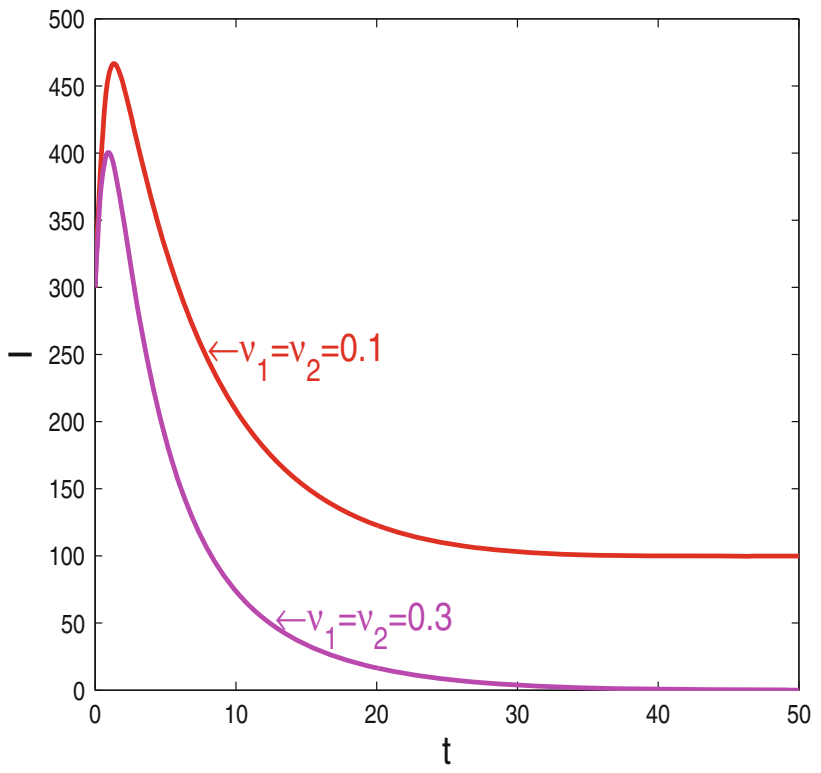

Fig. 6 Variation of infective population with time for different rates of treatment

cation as the case detection rate $\eta$ is quite high. Effect of rate of treatment is demonstrated in Fig. 6, where it is observed that by increasing the rate of treatment, disease can be eliminated from the population, i.e., with the increase in the rates of treatment, $\mathcal{R}_{0}$ decreases and the disease free equilibrium becomes stable.

\section{Conclusion}

Here a deterministic model for the transmission dynamics of TB in a population is designed and analyzed. It is shown that the model undergoes the phenomenon of backward bifurcation, when the associated reproduction number is less than unity. These results are very important as they govern the elimination and/or persistence of the TB disease in a community. The proposed model gives a clear picture of the impact of treatment and its relationship with case detection on the dynamics of tuberculosis. Overall, this study shows that the prospects of controlling the spread of TB is bright if only the treatment strategy can be sustained and case detection significantly improved upon.

Acknowledgments This research was supported by the research Grants of DST, Govt. of India, via a sponsored research project: SR/S4/MS:681/10.

\section{References}

1. WHO (2011) Global Tuberculosis Control 2011,WHO, Geneva, 9. www.who.int/tb/publications/global_report/ 
2. Humphreys H (2007) Control and prevention of healthcareassociated tuberculosis: the role of respiratory isolation and personal respiratory protection. J Hosp Infect 66:1-5

3. Magombedze G, Garira W, Mwenje E (2006) Mathematical modeling of chemotherapy of human TB infection. J Biol Syst 14:509553

4. Naresh R, Pandey S, Shukla JB (2009) Modeling the cumulative effect of ecological factors in the habitat on the spread of Tuberculosis. Int J Biomath 2:339-355

5. Bowong S, Emvudu Y, Moualeu DP, Tewa JJ (2010) Mathematical properties of a Tuberculosis model with two differential infectivity and $\mathrm{N}$ latent classes. J Nonlinear Syst Appl :13-26

6. Okuonghae D, Aihie V (2008) Case detection and direct observation therapy strategy (DOTS) in Nigeria: its effect on TB dynamics. J Biol Syst 16:1-31
7. Hethcote HW (2000) The mathematics of infectious diseases. SIAM Rev 42:599-653

8. Van den Driessche P, Watmough J (2002) Reproduction numbers and sub-threshold endemic equilibria for compartmental models of disease transmission. Math Biosci 180:29-48

9. Castillo-Chavez C, Song B (2004) Dynamical models of tuberculosis and their applications. Math Biosci Eng 1:361-404

10. Feng Z, Castillo-Chavez C, Capurro AF (2000) A model for tuberculosis with exogenous reinfection. Theor Pop Biol 57:235-247

11. Lakshmikantham V, Leela S, Martynyuk AA (1989) Stability Analysis of Nonlinear Systems. Marcel Dekker, Inc., New York and Basel 